\title{
Can STOP Trial Velocity Criteria Be Applied to Iranian Children with Sickle Cell Disease?
}

\author{
Reza Bavarsad Shahripour, ${ }^{\mathrm{a}, \mathrm{b}}$ Martin M. Mortazavi, ${ }^{\mathrm{c}, \mathrm{d}}$ Kristian Barlinn, ${ }^{\mathrm{b}, \mathrm{e}}$ Bijan Keikhaei, ${ }^{\mathrm{f}}$ \\ Hadi Mousakhani, ${ }^{\mathrm{f}}$ Mahmoud Reza Azarpazhooh, ${ }^{\mathrm{g}}$ Morteza Oghbaee, ${ }^{\mathrm{a}}$ Seyed Aidin Sajedi, ${ }^{\mathrm{a}}$ \\ Jessica Kepplinger, ${ }^{\mathrm{b}, \mathrm{e}}$ R. Shane Tubbs, ${ }^{\mathrm{h}}$ Karen C. Albright, ${ }^{\mathrm{b}}$ Andrei V. Alexandrov ${ }^{\mathrm{b}, \mathrm{i}}$ \\ a'Department of Neurology, Ahvaz University of Medical Sciences, Ahvaz, Khuzestan, Iran \\ ${ }^{b}$ Comprehensive Stroke Center, Department of Neurology, University of Alabama at Birmingham, Birmingham, AL, USA \\ 'Division of Neurological Surgery, University of Alabama at Birmingham, AL, USA \\ dDepartment of Neurological Surgery, University of Washington, Seattle, WA, USA \\ eDresden University Stroke Center, University of Technology Dresden, Dresden, Germany \\ ${ }^{\mathrm{f}}$ Research Center for Thalassemia and Hemoglobinopathy Ahvaz Jundishapur University of Medical Sciences, Ahvaz, Khuzestan, Iran \\ 'Department of Neurology, Mashhad University of Medical Sciences, Mashhad, Iran \\ h'Division of Neurological Surgery, The Children's Hospital of Alabama, AL, USA \\ iDepartment of Neurology, The University of Tennessee Health Science Center, Memphis, TN, USA
}

Background and Purpose Sickle cell disease (SCD) is strongly linked to stroke across all haplotypes in the pediatric population. Transcranial Doppler (TCD) ultrasound is known to identify the highest risk group in African-Americans who need to receive and stay on blood transfusions, but it is unclear if the same flow velocity cut-offs can be applied to the Iranian population. We aimed to evaluate baseline TCD findings in Iranian children with SCD and no prior strokes.

Methods Children with genetically confirmed SCD (Arabian haplotype, homozygote) and without SCD (controls) were prospectively recruited from pediatric outpatient clinic over a period of 9 months. We performed TCD in both groups to determine flow velocities in the middle cerebral (MCA) and terminal internal carotid arteries (TICA).

Results Of 74 screened children, 60 met the inclusion/exclusion criteria (62\% female; mean age $10 \pm 4$ years). Baseline characteristics did not differ between the cases and controls, except hemoglobin $(\mathrm{Hb})$ which was significantly lower in the SCD group $(P<0.001)$. The right MCA TAMM (Time Averaged Maximum Mean) was significantly higher than in controls (125+ $5.52 \mathrm{~cm} / \mathrm{s}$ vs. $92.5+1.63 \mathrm{~cm} / \mathrm{s}, P<0.001)$. Left MCA did not show differences. The TICA TAMM was also different between cases and controls $(P<0.05)$.

Conclusions Among Iranian children with asymptomatic SCD and without receiving recent transfusion TCD velocities are higher as compared to healthy controls but appear much lower than those observed in STOP (Stroke Prevention Trial in Sickle Cell Anemia) studies. We hypothesize that some children at high risk may be present with velocities lower than 170-200 $\mathrm{cm} / \mathrm{s}$ thresholds. A prospective validation of ethnicity-specific prognostic criteria is warranted.
Correspondence: Andrei V. Alexandrov Semmes-Murphey Professor and Chairman, Department of Neurology, The University of Tennessee Health Science Center, 855 Monroe Avenue, Suite 415 Memphis, TN 38163, USA Tel: +1-901-448 6199

Fax: +1-901-448 7440 E-mail: avalexandrov@att.net

Received: February 27, 2014

Revised: April 28, 2014

Accepted: April 28, 2014

The authors have no financial conflicts of interest.

Keywords Sickle cell; TCD; STOP criteria

\section{Introduction}

Sickle-cell disease (SCD) is a common genetic disorder af- fecting about $5 \%$ of the world's population..$^{1-4}$ It is particularly common among African and Arabian populations. ${ }^{1,3,5}$ Although SCD is strongly linked to stroke across all haplotypes in the pe- 
diatric population, Iranian children with a Saudi Arabian aplotype are less affected by this complication. ${ }^{6.9}$

Transcranial Doppler (TCD) is known to identify the highest risk group in African-Americans who need to receive and stay on blood transfusions. However, it is unclear if the same flow velocity cut-offs can be applied to Iranian children. ${ }^{10,11}$ Therefore, we performed a case-control study on prospectively collected data and aimed to evaluate baseline TCD findings in Iranian children with SCD and no prior strokes.

\section{Methods}

Children with genetically confirmed to be homozygote Saudi Arabian SCD (experimental group) and children without SCD (controls) were prospectively recruited from our pediatric outpatient clinic over a period of 9 months (04/2010-02/2011). Inclusion criteria were as follows: 3-16 years of age, hemodynamic stability and freedom from any medication that affects cerebral hemodynamics such as hydroxyurea and similar immunomodulating agents. Exclusion criteria: blood transfusion within 3 months prior to day of visit, history of brain trauma in the 3 months prior to their visit, history of previous stroke or evidence of pre-existing stroke on brain imaging, significant systemic disease (e.g. fever, chronic obstructive pulmonary disease, severe asthma, renal failure, congestive heart failure, uncontrolled hypertension, uremia, liver cirrhosis), other brain pathologies (e.g. brain tumors or infectious lesions), history of seizure disorder requiring anticonvulsant therapy, history of prenatal or perinatal hypoxic-ischemic brain injury, or evidence of human immunodeficiency virus infection. All patients were free of a neurological deficits.

Hemoglobin $(\mathrm{Hb})$ electrophoresis was performed to identify participants with $\mathrm{HbS}$. If positive, those children underwent confirmatory genetic testing for SCD. DNA samples were extracted from peripheral red blood cells following instructions on the available commercial kits (Qiagen W, USA). The analysis of $\beta^{S}$ haplotypes was carried out using PCR-restriction fragment length polymorphism. IRB approval was obtained from parents who gave written informed consent to participate in this study.

Transcranial Doppler was performed within 14 days of the hematologic work-up and independently of clinical data by an experienced sonographer blinded to clinical data. 2-MHz, power-motion Doppler (MultiDop X4 system, DWL, Germany) was used with application of a standardized insonation protocol. We used the transtemporal approach for insonation of the middle cerebral (MCA) and the terminal internal carotid artery (TICA). After sonographers optimized the waveforms, the highest peak systolic (PSV), end-diastolic (EDV), and time-av- eraged maximum mean (TAMM) velocities for the TICA and MCA were obtained from the TCD automatic tracing software. Using the STOP (Stroke Prevention Trial in Sickle Cell Anemia Study) criteria, TAMM was classified as normal (TAMM $<170$ $\mathrm{cm} / \mathrm{sec}$ ), conditional (TAMM 170-199 cm/sec), and abnormal ( $\mathrm{TAMM} \geq 200 \mathrm{~cm} / \mathrm{sec}$ ).

\section{Statistical analysis}

Statistical analysis was carried out using PASW Statistics 18.0 (SPSS Inc., Chicago, IL). Proportions were compared using Pearson Chi-squared or Fisher's exact test where appropriate. Measures of central tendency were compared using independent-samples Student $\mathrm{t}$ test or Mann-Whitney U test, where appropriate. A $P$ value less than 0.05 was considered as significant.

\section{Results}

Of 74 screened children, 60 children met the inclusion/exclusion criteria ( $62 \%$ female; mean age $10 \pm 4$ years, range 3-16 years). Genotype testing revealed Saudi Arabian haplotype ( $\beta^{\mathrm{S}}$ / $\left.\beta^{S}\right)$ in 34 patients. Baseline characteristics did not differ between the cases and controls, except hemoglobin $(\mathrm{Hb})$, which was significantly lower in the SCD group (mean $8.6 \pm 1.43 \mathrm{~g}$ / dL vs. $10.3 \pm 1.03 \mathrm{~g} / \mathrm{dL}, P<0.001$; Table 1$)$.

In children without SCD, the highest TAMM was $118 \mathrm{~cm} / \mathrm{s}$ (range 74-118 cm/s). Among children with SCD, 73\% had TAMM higher than the maximum value seen in controls (range $73-210 \mathrm{~cm} / \mathrm{s})$. The right MCA TAMM was significantly higher than in controls $(125+5.52 \mathrm{~cm} / \mathrm{s}$ vs. $92.5+1.63 \mathrm{~cm} / \mathrm{s}, P<$ $0.001)$. Left MCA did not show differences $(107.5+6.6 \mathrm{~cm} / \mathrm{s}$ vs. $99+0.56 \mathrm{~cm} / \mathrm{s}, P=0.65)$. The TAMM of TICA was also different between cases and controls (right: $79+2.8 \mathrm{~cm} / \mathrm{s}$ vs. $70+1.4 \mathrm{~cm} / \mathrm{s}$ and left: $78+2.89 \mathrm{~cm} / \mathrm{s}$ vs. $72.5+0.95 \mathrm{~cm} / \mathrm{s}$, respectively; $P<0.05$, Figure 1$)$. There were no TAMM differences between genders (Table 2). Among Iranian children with SCD only $1 / 30(3 \%)$ had conditional $>170 \mathrm{~cm} / \mathrm{s}$ and one patient (3\%) had abnormal ( $\geq 200 \mathrm{~cm} / \mathrm{s})$ TAMM velocities by the STOP trial criteria.

Table 1. Baseline Characteristics

\begin{tabular}{lccc}
\hline & $\begin{array}{c}\text { Controls } \\
\mathrm{N}=30\end{array}$ & $\begin{array}{c}\text { Cases } \\
\mathrm{N}=30\end{array}$ & Pvalue \\
\hline Age, mean (SD) & $10.3( \pm 3.1)$ & $9.7( \pm 4.0)$ & 0.528 \\
Female, \% & 67 & 60 & 0.592 \\
Hb, median (range) & $8.6(7.2-10.0)$ & $8.6(6.0-12.0)$ & 0.851 \\
TAMM R MCA, median (range) & $92.5(74-111)$ & $125(75-185)$ & $<0.001$ \\
TAMM L MCA, median (range) & $99(82-118)$ & $107.5(73-210)$ & 0.65 \\
TAMM R TICA, median (range) & $70(46-81)$ & $79(60-118)$ & $<0.001$ \\
TAMM L TICA, median (range) & $72.5(63-83)$ & $78(60-168)$ & $<0.001$
\end{tabular}



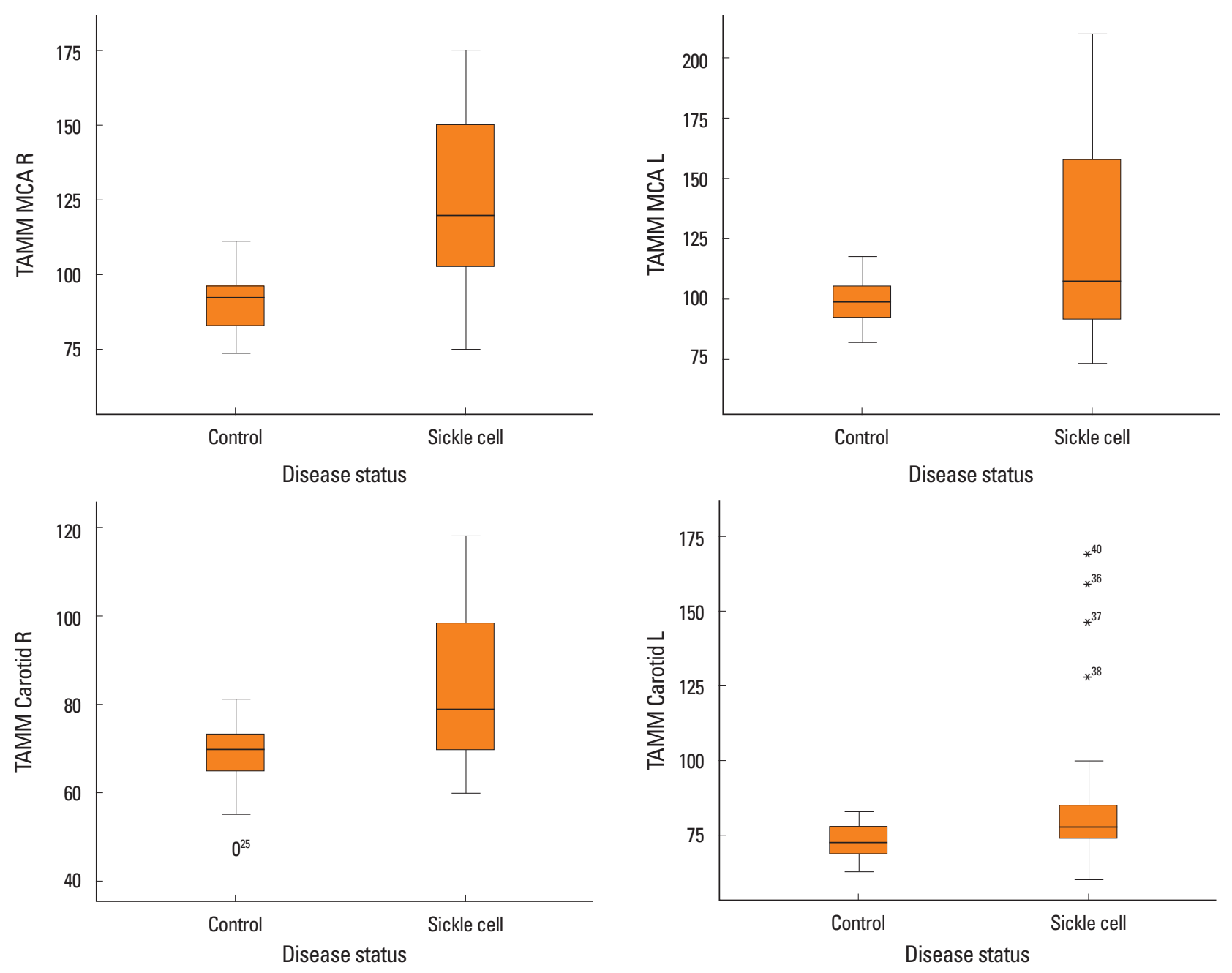

Figure 1. Comparison of Ultrasound Findings in Sickle Cell Patients and Controls.

Table 2. Gender Specific Ultrasound Findings in SCD Patients

\begin{tabular}{lccc}
\hline & $\begin{array}{c}\text { Males } \\
\mathrm{N}=12\end{array}$ & $\begin{array}{c}\text { Females } \\
\mathrm{N}=18\end{array}$ & $P$ value \\
\hline Hb, median (range) & $8.6(7.2-10.0)$ & $8.6(6.0-12.0)$ & 0.851 \\
TAMM R MCA, median (range) & $105.00(75-175)$ & $135.00(78-185)$ & 0.043 \\
TAMM L MCA, median (range) & $105.50(74-169)$ & $110.50(73-210)$ & 0.491 \\
TAMM R TICA, median (range) & $80.00(65-118)$ & $79.00(60-110)$ & 0.415 \\
TAMM L TICA, median (range) & $78.00(60-100)$ & $79.50(65-168)$ & 0.573 \\
\hline
\end{tabular}

\section{Discussion}

Sickle cell disease is caused by a mutation in the gene coding for beta globine chain, and is transmitted in an autosomal recessive fashion, which means that an individual needs to inherit the defective gene from both parents to develop the disease. An individual with only one copy of the defective gene (heterozygous carrier) is clinically asymptomatic (sickle cell trait).

Early investigators noted that SCD patients in the United States were almost always of African origin. ${ }^{12}$ Subsequent global epidemiological studies established that SCD and sickle-cell trait are common in parts of sub-Saharan Africa, the Saudi Arabian peninsula, central India, and certain parts of Southern Europe. ${ }^{13}$

By using Restriction Fragment Length Polymorphism (RFLP) analysis, geneticists have found many distinct haplotypes. ${ }^{13}$ Five distinct $\beta$-globin haplotypes are reported in patients with sicklecell anemia. These are Benin, Cameroon, CAR/Bantu, Saudi Arabian, and Senegal. Indeed, these hasplotypes are not restricted to the eponymous nation, and they can be found broadly distributed. ${ }^{13}$

The haplotypes of African-American sickle-cell patients correspond to locations of forced African emigration to the New World through the African slave trade. In a study of 98 SCD patients from the state of Georgia, 54\% had the Benin haplotype, $27 \%$ had the Bantu haplotype, and $15 \%$ had the Senegal haplotype. ${ }^{14}$ Sickle cell disease is also highly prevalent Arab states. Some of the world's highest frequencies of the disease are seen in Saudi Arabia (5.2\%), Oman (3.8\%), and UAE (1.9\%). Lower values are observed in Yemen (0.95\%), Bahrain (0.7\%), and 
other Arab countries. ${ }^{15}$ Studies in population genetics have revealed that the extent of clinical severity in sickle cell disease (from mild to severe) is related to the genetic background (haplotype) of the affected individual. ${ }^{15}$

Recent anecdotal observations among the Saudi / Arabia and Senegal haplotypes suggest they are associated with milder forms of SCA, whereas the Bantu haplotype is associated with a more severe clinical course. ${ }^{16}$ Interestingly, this observation correlates with differences in the mean levels of fetal hemoglobin (FH) in patients with different haplotypes. Individuals with the Saudi/Arabia haplotype had an average $17 \% \mathrm{FH}$, those with the Senegal haplotype had an average $12.4 \% \mathrm{FH}$, and those with the Bantu and Benin haplotypes had even lower average FH. However, within each haplotype group, the range of $\mathrm{FH}$ levels is very broad. ${ }^{4,17,18}$

On the other hand, Iranian children are a natural reservoir of SCD due to high frequency of the Sickle gene in the Arabic subpopulation of Iran (2-15\%).,18 Clinical Practice of medicine has shown that the prevalence of sickle cell disease is high in the southern part of Iran, especially in Khuzestan province at the border of Iraq and Persian Gulf. This may be due to the ethnic Arabian Iranians who live in this region. ${ }^{17}$

Children with SCD are 200 to 400 times more likely to suffer a stroke, compared to children without SCD. Approximately $10 \%$ of African-American patients have a clinical stroke prior to 20 years of age and another $22 \%$ have a silent infarction detectable on magnetic resonance imaging. Stroke has also been described in Mediterranean and African SCD patients although with lesser frequencies ( $4.1 \%$ and $6.7 \%$, respectively). ${ }^{3,14}$

Blood transfusions with normal RBC can prevent a first stroke when the TCD is abnormal, and they can generally prevent second and third strokes in patients with SCD who have already had a stroke. ${ }^{14}$

In 1990, a large national study called the Stroke Prevention Trial in Sickle Cell Anemia (STOP) demonstrated that children with abnormal TCD have a $90 \%$ lower risk of stroke with monthly blood transfusion. Therefore, an annual TCD for SCD-patients is recommended; beginning at 24 months of age, this should be repeated every 6-12 months during early childhood. Currently, TCD is the only recommended method for treatment for primary-stroke prevention. Indeed, the clinical valid prognostic tool available for primary stroke prevention is periodic screening of time-averaged mean velocities (TAMVs) in the distal internal carotid arteries and middle cerebral arteries using TCD. ${ }^{4}$

Children with abnormal TCD, defined as TAMV $\geq 200 \mathrm{~cm} /$ $\mathrm{s}$, have an approximately 44 -fold greater risk of developing primary stroke than those with normal TCD velocities (TAMV
$<170 \mathrm{~cm} / \mathrm{s}$ ). As we mentioned before, in this group, blood transfusion decreases the risk of stroke by more than $90 \% .{ }^{1,14}$ Our study showed that among Iranian children with asymptomatic SCD and without a recent blood transfusion, velocities are greater compared to healthy controls but very few have conditional and abnormal velocities observed in the STOP trials. We hypothesize that Iranian children at higher stroke risk may be identified with TAMM velocities below $170 \mathrm{~cm} / \mathrm{s}$.

In the STOP trial, only one stroke was reported in the treatment group (received transfusions) compared to 11 cases of stroke in the control group (did not receive transfusion, and had high cerebral circulation velocity) at 20 months follow up. ${ }^{1,14,19}$ But in our study, at one year follow up, no cerebrovascular accidents were seen. It appears that our patients had a lower incidence of stroke with no transfusion or any prophylactic treatment.

Since this study was the first study evaluating the velocity changes in a race-specific haplotype (Saudi Arabian), it appears that haplotype might play a pivotal role in the development of the disease. Hence, we propose that STOP criteria be revised depending on race and haplotype. We believe that meaningful differences in MCA TAMM and ICA TAMM in STOP might be due to differences in genetic haplotype of our patients compared to African- Europe haplotype.

Our study is the first to report TCD findings in Iranian children with SCD. It also has some limitations including relatively narrow range of ages and relatively small sample. Furthermore, we excluded patients with SCD and stroke symptoms and those already receiving transfusions; subjects that could have yielded different velocity values. Nonetheless, our report questions whether the STOP trial velocity criteria are applicable to Iranian children with SCD. In our study, only 2 out of 30 patients (6\%) with genetically confirmed Saudi Arabian haplotype had conditional or abnormal flow velocities. This is less than in the STOP trial (10\% to $15 \%))^{1,19}$ Although this finding parallels the lower incidence of stroke in an Iranian SCD population, ${ }^{9}$ it does not necessarily mean that STOP velocity criteria should be automatically applied to a different genetically-driven population at risk.

\section{Conclusions}

We conclude that STOP criteria need to be revisited to determine whether the same or adjusted velocity criteria should be used in different ethnicities and haplotypes of SCD. A prospective validation study of ethnicity-specific prognostic criteria is warranted. 


\section{References}

1. Ohene-Frempong K, Weiner SJ, Sleeper LA, Miller ST, Embury S, Moohr JW, et al. Cerebrovascular accidents in sickle cell disease: rates and risk factors. Blood 1998;91:288-294.

2. Lobo CL, Cancado RD, Leite AC, Dos Anjos AC, Pinto AC, Matta AP, et al. Brazilian Guidelines for transcranial doppler in children and adolescents with sickle cell disease. Rev Bras Hematol Hemoter 2011;33:43-48.

3. Huttenlocher PR, Moohr JW, Johns L, Brown FD. Cerebral blood flow in sickle cell cerebrovascular disease. Pediatrics 1984;73:615-621.

4. Adams RJ. Stroke prevention and treatment in sickle cell disease. Arch Neurol 2001;58:565-568.

5. Zandian K, Keikhaie B, Pedram M, Kianpoor Ghahfarokhi F. Prenatal diagnosis and frequency determination of alpha and beta thalassemia, S, D, C, and $\mathrm{H}$ hemoglobinopathies; globin mutational genes aanalysis among voluntary couples from Ahvaz. Iran J Blood Cancer 2009; 1:95-98.

6. Ahmadzadeh A, Moghtaderi M, ZandianNeonatal K. Screening for Sickle Cell Disease in South West Iran: a Pilot Study. Iran J Blood Cancer 2011;3:117-120.

7. Zandian K, Pedram M, Kianpour Ghahfarokhi F. Pre-marriage sickle cell screening program in south region of Iran, a pilot study on 50 cases of sickle trait. Iran J Blood Cancer 2009; 1:55-57.

8. Haghshenass M, Ismail-Beigi F, Clegg JB, Weatherall DJ. Mild sickle-cell anaemia in Iran associated with high levels of fetal haemoglobin. JMed Genet 1977;14:168-171.

9. Amarenco P, Bogousslavsky J, Caplan LR, Donnan GA, Hennerici MG. New approach to stroke subtyping: the A-S-C-O (phenotypic) classification of stroke. Cerebrovasc Dis 2009;27:
502-508.

10. Barnes MG, Komarmy L, Novack AH. A comprehensive screening program for hemoglobinopathies. JAMA 1972;219: 701-705.

11. Russell MO, Goldberg HI, Hodson A, Kim HC, Halus J, Reivich $\mathrm{M}$, et al. Effect of transfusion therapy on arteriographic abnormalities and on recurrence of stroke in sickle cell disease. Blood 1984;63:162-169.

12. Caplin I, Haynes JT, Lloyd F. Incidence and significance of sickle cell trait in asthma. Ann Allergy 1972;30:623-626.

13. Gabriel A, Przybylski J. Sickle-cell anemia: a look at global haplotype distribution. Nature Education 2010;3:2.

14. Hattori Y, Kutlar F, Kutlar A, McKie VC, Huisman TH. Haplotypes of beta $S$ chromosomes among patients with sickle cell anemia from Georgia. Hemoglobin 1986;10:623-642.

15. Jastaniah W. Epidemiology of sickle cell disease in Saudi Arabia. Ann Saudi Med 2011;31:289-293.

16. Steinberg MH. Predicting clinical severity in sickle cell anaemia. Br J Haematol 2005;129:465-481.

17. Miller BA, Olivieri N, Salameh M, Ahmed M, Antognetti G, Huisman TH, et al. Molecular analysis of the high-hemoglobin-F phenotype in Saudi Arabian sickle cell anemia. N Engl J Med 1987;316:244-250.

18. Labie D, Pagnier J, Lapoumeroulie C, Rouabhi F, Dunda-Belkhodja O, Chardin P, et al. Common haplotype dependency of high $\mathrm{G}$ gamma-globin gene expression and high $\mathrm{Hb} \mathrm{F}$ levels in beta-thalassemia and sickle cell anemia patients. Proc Natl Acad Sci USA 1985;82:2111-2114.

19. Adams RJ, McKie VC, Brambilla D, Carl E, Gallagher D, Nichols FT, et al. Stroke prevention trial in sickle cell anemia. Control Clin Trials 1998;19:110-129. 